\title{
Index
}

Abbot-Anderson, Sir Maurice 191, 206n.72, 207n.94

abortifacient 118

adultery 9o, 111, 125, 128n.57, 178-9, 198

Afraid of Love film 200

Ahmed, Leila 146, 169n.91, n.93, n.95, 17on.111, 172n.150

Aitken, Bessie 107-9, 113, 115, 117, 123, 125

alcohol 5, 55, 56, 60, 63-4, 109

licensing hours 61,64

Alexander, Sally 11, 94n.66, 121, 127n.46, 128n.74, 131n.140, 207n.108, 209n.154

Alibert, Raymonde 142

Alibert, Yvonne 146, 167n.33

Allatini, Rose 53n.168

aliens $38-40,42-4,67,71-2,79,85,98$ n.16o, 204n.5

Allan, Maud 15-44, 52n.150, 53n.183, 63, 113 , $150,156,167$ n.43, 178-9, 210, 213, 215-17

Allen, Judith A 13on.123

Ampthill, Lady Margaret 181, 195, 204n.23, 205n.53

Ampthill. Lord Oliver 178-9, 203n.5, 212 anti-semitism 17, 39, 40 see also Jews and Jewishness

Arabian or Thousand and One Nights 156-7 aristocrats and aristocracy 23, 47, 83, 92n.43, $161,176,186,213$

Asquith, Cynthia 40, 43, 49n.85, 5on.122, $51 \mathrm{n} .126$

Asquith, Herbert Henry 16, 19, 21-3, 29, $32-3,40-1,45$ n. 6 , 50n.97, 210

Asquith, Margot 16, 17, 19, 22-4, 27-9, 32-3,
40-1, 43-4, 47n.49, 48n.50, 49n.85, 210 Attorney General 196

Auerbach, Sascha 83, 92n.39, 95n.86, 96n.104, n.108, 99n.168, n.183, 10on.188, n.189, 218n.3

'bachelor girls' 3 see also flapper

Ballets Russes 21, 156

Ballinger, Anette 117, 130n.107, n.109, 150, 170n.118, 213, 219n.17

Baroudi, Mahmoud 147-8

Baxter, Beverley 112, 128n.68, 195

Beaverbrook, Lord (Max Aitken) 8-9

Beddoe, Deirdre 7, 11n.13, 13n.31, 126n.21, 127n.48, n.49

Bell, Anne Olivier 11n.1, 47n.49

Belloc Lowndes, Marie 16, 45n.9, 47n.47, 48n.50, n.56, n. 58,52 n. 154

Bennett, Arnold 38, 51n.141, 166n.6

Body and Soul 183, 205n.35

Bernhardt, Sarah 45n.7

Berridge, Virginia 91n.12, n.14, 95n.88, n.98 Billing, Noel Pemberton 15-19, 23-44, 46n.16, n.18, 5on.122, 51n.130, n.141, $53 \mathrm{n} .165,144,217$

Bingham, Adrian 3, 7, 11n.12, 12n.14, n.15, n.20, 13n.33, 14n.47, 49n.82, 94n.78, 98n.154, 127n.38, 169n.79, 200, 203n.4, 204n.19, 209n.140, n.146, 219n.19, n.21

Bioscope 200, 202, 209n.148, 150

Bishop of London 200

'Black Book' 15-16, 32-5, 38-40, 5on.118, 52n.163 
Blecourt, Willem de 206n.82

Bodkin, Sir Archibald 142-3

book-reading 112-13, 116, 125

Boone, Joseph 144, 168n.72, 171n.136, n.138, 173 n. 160

Bottomley, Horatio 38, 42, 53n.177

Bovary, Madame 115, 116, 129n.84, 214

Bow Street police court 25, 49n.81, 85, 134, 139, 166n.13

Braybon, Gail 12n.17, n.19, n.24

Breward, Christopher 93n.60, 114, 129n.83

birth control 187

birth rate, British 110

Britishness 72, 216-17 see also Englishness

Brittain, Vera 3, 11n.7

Broken Blossoms 73-74, 88, 97n.128

buggery 140

see also sodomy

Burke, Thomas 73, 80, 95n.97, 97n.123, n.126, n.129, n.130, 99n.170, n.171, n.179, $173 n .154$

Burton, Richard, 153, 171n.136, 172n.153 'Sotadic Zone' 153

'butterfly woman' $6,56-7,59-62,88-9$, 91n.12, 93n.46, n.54, n.55, 144, 213-14, 217

Bywaters, Freddy 1, 102-6, 108, 111-12, 116-19, 120, 122-4, 126n.12, 128n.69, n.71, n.75, n.76, 144, 147, 204n.8

Caillaux, Madame Henriette 22-3

Cairns, J.A.R. 74, 76-7, 88, 98n.139, n.145, 211

Campbell, Mrs Patrick 116

capital punishment 104, 122, 124, 125n.7, 127n. 55, 131n.147, 211, 217, 219n.7

Carleton, Billie 57-63, 69, 81-3, 92n.23, 166n.13, 210

Carlton and Prior 108

Carnarvon, Lord (George Herbert) 152

Carpenter, Edward 157

Carter, Howard 152

Carter Wood, John 7, 13n.36, n.45, 219n.11 Castlehaven, Lord (Mervyn Touchet) 153

Cecil, Hugh 202

celibacy 194, 208n.112

censorship 9, 36, 140, 167n.47, 178, 215, 216
Central Criminal Court see Old Bailey

Chang, Brilliant 65-9, 77, 81, 89, 210

Chapman, Revd Hugh 119, 123

Chepstow, Ruby 148

Childers, Erskine 38, 52n.154

Chinese laundry 71, 73, 96n.104

Chinese 10, 56, 59, 65, 67-74, 76-83, 85-90, $94 \mathrm{n} .83,96 \mathrm{n} .104$, n.126, 217, 218n.2

'Chinaman' 56, 65-9, 73-4, 76-7, 79, 82, 86-9, 96n.114, 99n.168

as good husbands $76-8,97$ n.131, 98 n.145

Chow, Karen 16o, 174n.168, n.173, n.175

Churchill, Robert 138-9, 145, 169n.85

Clarke, Edward 136, 166n.21

Clarke, Dr J.H. 26

Clarke, Percival 136-7, 141-5, 148-51, 154, 165 clairvoyant 178, 189, 190, 206n.82, 207n.84 clitoris $25-7,36-7,187$

cult of $15,17-18,22,25,44,210,215$

cocaine $57-62,64-8,77,81,83,88$, 91n.12, n.13, n.14, 92n.23, 99n.179, 112

known as 'snow' 64, 88, 94n.71

Cohler, Deborah 37, 46n.10, 52n.149

Conan Doyle, Sir Arthur 152, 207n.88

contraceptives 206

Conquest, Joan 159, 161, 174n.18o, n.181, n.189

Cook, Hera 209n.153

Cook, Matt 126n.9, 171n.132

Cooke, Dr Serrell 25-9, 31

Cooper, Lady Diana, née Manners 19, 22, $36-7,47 \mathrm{n} .35,58,120-1,202$

Cooper, Duff 28, 36-7

Corelli, Marie 18, 152

co-respondents 164, 179, 181, 196-7, 200, 203n.10, 204n.36, 208n.134

Court of Appeal 200

cosmopolitanism 21, 23, 41, 44-5, 136

Criminal Evidence Act (1898) 138

Criminal Justice Act (1925) 122

Crippen, Dr Henry Harvey 15, 45n.2, 129n.82 Cromer, Lord (Evelyn Baring) 146 cross-dressing 10, 102, 125n.2, 176, 184, 186, 205n.46, n.49

Curtis-Bennett, Henry 103-4, 111, 118, 136

'dailies' 2, 8, 9, 160, 176, 215

Daily Chronicle 8, 119, 133, 137, 145, 149 
Daily Express 7, 8, 25, 29, 31, 33-4, 37, 43, 45n.6, 49n.86, 62, 64, 66-7, 69, 102, $106-8,112,118,140,154-5,159,164,183$, $195,198-9$

Daily Graphic 74-6

Daily Mail 7-8, 36, 38-40, 60, 70-1

Daily Mirror 3, 8, 35, 104, 105, 119, 163, 177, $184,187,215$

Daily Sketch 8, 58, 103-4, 111, 122-3, 134, 135, 147,159

Dacre Fox, Norah 42

dance $6,9,15-17,19,21,23,25,29,46$ n.27, 57, $61,63,65,89,93 n .62,106-8,112$, 127n.32, 156, 179, 181-2, 196, 198, 204n.25, 210, 213-14, 217

craze $21,45,63,108,213$

dance-hall 61, 63-4, 88, 93n.58, 106-8 instructresses 61-3, 92n.40, n.42, 93n.54

Darling, Charles 16, 27, 29, 34-5, 38, 42, 44, 48n.73, 5on.97, 51n.126, n.127, n.139, 52n.151, 53n.170, 55, 130n.112, 215

Darwin, Leonard 78

Das, Santanu 95n.103, 100n.192

Dawson, Jill 211, 219n.9

D'Cruze, Shani 129n.82, 130n.127, 17on.115, 175n.195

Defence of the Realm Act (DORA) 5, 12n.23 Regulation 4ob 57, 67, 81

Degen, Revd 5, 12n.20, 55, 76

Delafield, E.M. 114, 128n.59, 129n.82 desert 148, 157-8, 161, 164, 173n.157

Desert Love 161, 174n.18o, n.189

desert romances 6, 116, 139, 157-9, 161-4, 173n.163, 174n.181, 214, 218

de Veulle, Reggie 59, 63, 8o-2, 92n.23

Diaghilev's Russian ballet see Ballet Russe

divorce $2,3,7,10,86,111,133,142,146,162$, 164-5, 168n.56, 169n.91, 176, 178-81, 183 , $188,197-200,202-3,212,214,216$

Divorce and Matrimonial Causes Act (1857) 111

Doan, Laura 3, 11n.12, 16, 45n.10, 46n.14, 126n.11, 171n.134, 205n.52

Dodd, Dr Stanley 189, 191-2 dope 59, 62-4, 88, 90, 214

'dope fiend' 6, 56, 81, 89-90, 91n.12, 94 n.75, 10on.209 'dope decoy' 86

drugs $6,10,56-60,62-5,67,69,74,76,80-1$, $83,86,88-90,91 n .14$, n.19, 94 n.74 10on.205, 144, 214, 216

see also cocaine; opium

Douglas, Lord Alfred 29, 31, 40-1, 49n.86, 5on.99, n.107

Douglas, James 63, 93n.54, 111-12, 114-16, 119 , 13on.105, 131n.130, 158, 163, 17on.163, 214

Duke, Sir Henry 187-8, 200, 209n.145

Duncan, Isadora 19, 47n.32

Durrant, Theo 19

Dyhouse, Carol 126n.17, 127n.38

Eden, Dr Thomas 189, 191-2, 194, 207n.91, n.96

Egypt 3, 116, 132-3, 138-9, 144, 146, 151-3, 155,16 o-1, 164, 166n.3

Egyptian 1, 90, 132, 140-3, 146-9, 151-3, 156-7, 164-5, 167n.34, 169n.91, 173n.157, 213

Egyptian feminism 169n.91, 170n.111

Ellis, Havelock 49n.79, 205n.49

Empire News 61-2, 64, 67-9, 71, 85-6, 88-9, $102,109,144,147,186$

Enani, Said 133, 137-8, 141-3, 146, 167n.34, n. $37,168 \mathrm{n} .58$

Englishman 86, 152, 164, 184

Englishness 32-3, 43-4, 72, 114, 217

eugenics 78, 110

Eugenics Society 78

Evening News 8, 39, 65, 68, 74, 76, 137

Fahmy, Ali 1, 132-53, 162, 167n.34, n.47, 171n129, 213

Fahmy, Marguerite 1, 10, 132-51, 153, 155-7, 162-3, 166n.5, 167n.27, n.33, n.37, n.41, 168n.56, 173n.157, 179, 210-14, 217-18

'fashionable' women 88, 119, 124, 136, 154-6, 162,180

Felski, Rita 126n.13, 129n.8o

femme fatale 118, 123-4

feminist 12n.23, 41-3, 65, 99n.162, 104, 122, 129n.94, 143, 146, 208n.112 see also suffragettes

First World War see Great War 
Fisher, Kate 192, 207n.100, n.103, n.108 flapper $3-4,6,10,45,55-7,60,63,106-8$, 126n.21, 158, 161, 164, 180, 182, 202, 213, 217-18

see also modern woman

Fleming, Rachel 78-9, 98n.156

Foreign Office 72, 77, 97n.127, 98n.148

Forster, E.M. 157

43 Club 61

Frigidity 194

fur, wearing of $66-7,107,119-20,126$ n.17

Gallichan, Walter 194, 208n.112, n.113 gambling 56, 69, 73-4, 76, 80, 87, 89, 108 Gates, Reginald Ruggles 188, 206n.70 gender inversion 102, 184

George V 178, 198, 216

Germans and Germany 15-18, 23, 27, 31-4, 36-44, 45n.6, n.7, 52n.154, n.163, 53n.165, n.167, n.169, n.181, 58, 6o, 119, $153,180,184,207$ n. 88 see also aliens

Gilman, Sander 45n.7, 49n.78, 208n.119

Gordon, Dr Edward 140, 168n.48

Gordon, Eleanor and Gwyneth Nair 7, 13n.36, 128n.67, 131n.135

Grand Guignol 147, 170n.97

Graves, Robert and Alan Hodge 12n.15, 93n. $58,109,127$ n. 38 n.45

Graydon, William 108, 123, 129n.85

Grayzel, Susan 12n.24, 13n.30, 48n.62

Great War 2, 7, 63, 70, 132, 156, 173n.64, 189 aftermath of $2,45,57,102,110,125,217,218$

Grein, Jack T. 32, 51n.127

Griffith, W.D. 73, 97n.129

Gullace, Nicoletta 12n.17, 42, 53n.176, n.178

Haldane, Richard 16, 33, 45n.6

Hall, Lesley A. 128n.56, n.74, 13on.112, 173n.164, 206n.7o, 207n.109

Hall, Radclyffe 5on.118, 112

Halperin, David 216, 219n.22

Hammersmith Palais de Danse 61, 92n.43

Hamilton, Cicely 104, 122, 124, 125n.4, n.7

harem 146-7

Hari, Mata 19, 25, 47n.29

Harrison, Austin 44, 51n.141
Hart, Blanche, Christabel Russell's mother 19o, 204n.25, 205n.53

Hassan, Ahmed Ben 160

Hastings, Patrick 182, 186, 191-2, 195-7, 206n.72, 208n.120, n.134

Heinberg, Rose 62, 65, 68

Herbert, A.P. (Alan Patrick) 198, 208n.135

heterosexuality $43,157,216-17$

'hidden hand', or 'unseen hand' 17, 38-9, 42, 52n.154, 60

Hichens Robert 157

Bella Donna 116, 147, 170n.99, n.100, 175n.189

The Garden of Allah 157, 170n.99

Hirschfeld, Magnus 153, 171n.134, 184

Hoare, Philip 16, 45n.4, n.10

Holloway prison 83, 210, 211

Home Office 40, 53n.167, 73, 79, 96n.115, 97n.127, 98n.147, n.148, n.158

homosexuality $17,29,37,40,44,49 n .93$, 53n.168, n.17o, 140, 153, 157, 184, 215-16 hormones 178, 190

Horwood, Catherine 109-10, 126n.17, 127n.44, 169n.79

Houlbrook, Matt 112, 113, 126n.9, 127n.38, 128n.70, n.72, 129n.95, 205n.48

House of Lords 179, 186, 200, 205n.56, 212

Huan, Cheng 73-4

Hull, E.M. (Edith Maud) 159-6o, 163, 174n.168. n.182. n.183

Hume-Williams, Ellis 29, 32, 35-7, 39, 43, 5on.107, 51n.126, 179, 182-3, 196-7, 200, 204n.9, 208n.133, 209n.147

Humphreys, Travers 18, 25, 46n.17, n.21, $52 n .151$

'Hunnish scenes' 187-8

Hunter, Eileen 198, 204n.7, n.10, n.24, 205n.56, 208n.125, 209n.137, n.138, 209n.14

hymen 176, 179, 187-9, 191-2, 203, 209n.156 Huyssen, Andreas 121, 129n.96, 131n.137

Hynes, Samuel 39, 53n.165, n.168, n.169, n.173

Ilford 103, 111, 114-15, 122

illegitimacy 5, 111

Illustrated Sunday Herald 9, 55, 86, 134, 157, $163,176,182$ 
incitement 103-4, 106, 117, 119, 122-5

Indian soldiers 5-6

inter-racial relationships $71-2,77,80-1,90$, $162-4$

Israel, Kali 202, 209n.152

Jackson, Holbrook 214

jazz 64, 94n.69, 161, 213

Jenkins, Huntly 59, 82, 92n.23

Jenkinson, Jacqueline 96n.105

Jews and Jewishness 39-42, 85

John Bull 9, 38-9, 53n.177, 88

Joynson-Hicks, William 39, 216

Judicial Proceedings (Regulation of

Reports) Act (1926) 200

jury 1, 34-5, 37, 39, 52n.151, 59, 104, 117, 119, $136,141-2,145,147-51,179-82,191,195$, 197-8

Kempton, Freda 57-8, 61-9, 94n.74, 117, 181, 210, 213

Kerner, Annette 58, 91n.17, 92n.21

Kettle, Michael 16

Kinematograph Weekly 202, 209n.149

Kingsley Kent, Susan 11n.4, 130n.123

Kipling, Rudyard 163

Kitt, Julia 88-90, 211, 215, 217

'khaki fever' 5-6

Kohn, Marek 58, 6o, 91n.19, 92n.20, n.23,

$$
\text { n.32, 95n.98, 99n.172, n.175 }
$$

Koritz, Amy 46n.28, n.29, 47n.31, n.41, n.46 von Krafft-Ebing, Richard 28, 31, 49n.92, n.94

Psychopathia Sexualis 28, 49n.92, 205n.49

'Lady Mab' 182-3, 197

Lawrence, T.E. 157

Leavis, Queenie 129n.94, 159, 174n.168

Le Queux, William 38, 52n.154

lesbian and lesbianism 9, 15-17, 22, 25-7, 29, $34-5,37,41,47$ n. 47, 49n.85, 215-16

Leslie, Dr Murray 57

letters 2, 38, 103-4, 110-13, 115-18, 121-2, 126n.21, 128n.61, n.68, 203, 208n.113, 211, 213, 215

Levine, Philippa 12n.24, 13n.26, 98n.134 Libel Act (1843) 18
Lidbetter, E.J. 78, 98n.151

Light, Alison 77, 98n.143, 114, 129n.81

Limehouse 56, 69-74, 76-83, 85-9, 97n.129, $98 \mathrm{n} .144,218 \mathrm{n} .2$

Liverpool 21, 70, 78-8o, 85-8, 96n.104, n.105, 97 n.126

Liverpool Courier 80

Lloyd George, David 22, 33

Lloyd's Sunday News 9, 107, 195, 197

Lord Chancellor 198

Lowndes, Marie Belloc see Belloc Lowndes, Marie

Macaulay, Rose 121

McClelland, Keith 13n.40, n.43, 14 n.47

McKibbin, Ross 93n.61, 126n.12, 127n.32, n. 44

McLaren, Angus 7, 13n.35, 45n.10, 49n.91, 5on.105, 125n.2, 207n.95 n.10o

make-up 109, 159, 214

Manchester Guardian 37, 121, 215

Manchu, Dr Fu 69, 73, 95n.97

Manners, Lady Diana see Diana Cooper

Marshall Hall, Sir Edward 136-48, 150-4, 157, 163, 166n.21, 167n.34, n.37, n.47, 171n.123, 179-8o, 183-4, 186-7, 190-9, 201, 205n.45, 206n.78, 208n.113, n.133, 216

Marlborough Police Court 81

Married Love 26, 48n.75, 113, 174n.168, 187-8, 203, 206n.69, 207n.101, 216 masculinity 27, 70, 158, 186-7, 196 maternity, see motherhood Matrimonial Causes Act (1923) 111 Matthews, Barrington 82 Matthews, Jill Julius 93n.58, n.61, 126n.13 Mayer, Edgar 179, 198, 200, 204n.10 Mayfair 59, 83, 85, 119, 183

Mead, Frederick 81-2

Medd, Jodie 16, 34, 46n.10, n.12, 51 n.128 Melman, Billie 7, 11n.13, 13n.31, 96n.115, 128n.74, 129n.89, 158, 161, 172n.145, 173n.161, n.163, n.165, 174n.168, n.172, 206n.64, 208n.124

melodrama 29, 32, 38, 52n.153, 118-19, 122, 13on.115, 144-5, 168n.73, 197, 213

Messalina 111, 128n.59

Metropolitan Police 36, 38, 94n.73, 168n.56 
Meyrick, Kate 61, 63, 65-66, 92n.40 n42, n. 43,93 n. 45 , n.55, n.59, 10on.198, 132, 166n.1, 210, 218n.2

Mills, Lady Dorothy 164, 175n.190, n.191 miscegenation 10, 72, 132, 134, 148, 162-3, 165,217

see also inter-racial relationships

Mitchell, Tim 147, 169n.94

Mitford, Nancy 212, 219n.13

Mixed Britannia 78-9, 98n.155, 99 n.162

Modern Girl Around the World Research Group $11 n .13$

Modernity 3, 6, 10, 11n.13, 22, 44-5, 55, 77, 9o, 104, 106-7, 109, 119, 121, 125, 126n.13, $156,165,181,184,195,207$ n. 88,208 n.112, $214,216,218$

modern woman $2-4,6-8,10,11 n .13,12 n .15$, $55-7,63,85,89-90,91 n .4,102,106-7$, $110,125,126 \mathrm{n} .21,165,176,178$, 191, $195-6,202,213-15,217-18$

Moody's nightclub 61 morality tales 90, 216 morbidity 117, 119-20, 123-4 Morning Post 8, 35, 43, 179

Morton, H.V. 152, 159, 173n.154 motherhood 7, 181, 195-6

Mothers' Union 200 munitions workers 4, 183, 196 Muskett, Herbert 81-2

Naismith, Mrs, clairvoyant 178, 190

National Archives 10, 140, 145

National Council of Women 200

nationalism 44, 72, 152

Egyptian 152

Irish 52n.154, 53n.17o, 152

Nava, Mica 11n.13, 47n.36, 50n.98, 128n.74, 172n.147

Nead, Lynda 13n.39, n.41, 97n.134, 122, 13on.126, 131n.144

Negri, Pola 131n.16o, 147

neurosis 6o, 117

News of the World 37, 62, 180, 215

New Statesman 3, 15, 36

New Survey of London Life and Labour 63, $129 n .89$
Nichols, Beverley 5on.99, 123, 127n.55, 13on.117, 131n.151, n.156, 161, 174n.179 nightclubs 6, 55-6, 61, 63-5, 67, 87-90, 93n.58, 94n.69, 73, 103, 181, 183, 196, 214 Normanton, Helena 136, 143, 166n.18 Northcliffe, Lord (Alfred Harmsworth) 8-9, $23,39,52 n .154$

Notable British Trials Series 10, 104, 211 nymphomania 27

Oddie, Samuel Ingleby 58-9, 166n.13

Old Bailey 1, 15, 22, 25, 27, 36-7, 59, 103, 106, $110,112,133-4,137,142,155-6$

Oosterhuis, Harry 49n.94, 5on.94 opium $57-60,67,69,73,77,80,81-3,85-8$, 99n.168, n.179, 211, 218n.4

den $81,83-4$, 99n.168

Oram, Alison 101n.210, 125n.2, 127n.49, 169n. 81

Orientalism 21, 23, 25, 32, 41, 44, 69, 73, 76, 78-9, 81, 132-4, 140-1, 143-4, 146-9, 151-3, 155-8, 162-5, 168n.68, 171n.138, $172 \mathrm{n} .153$

Owen, Harrison 115

Palace Theatre 19, 27, 47n.33, n.39

Panayi, Panikos 52n.156, n.157, 53n.18o, 96n.105

Pankhurst, Christabel 41-2, 53n.177

Pankhurst, Emmeline 42, 53n.177

Pelmanism 83

Pemberton Billing see Billing

People 9, 58, 134, 152, 156-7, 163-4, 182, 213, 215

Ping You, Ada 59, 80-3, 85-6, 90, 210-11, 217 as 'high priestess' 82

Ping You, Lsu 83, 211

A Pin to See a Peepshow 211, 218n.5

Poiret, Paul 21, 23, 48n.6o, 156

poisoning 7 , 92n.23, 103, 112, 116-18, 122, 128 n. $67,139,147-8,199$

Priestley, J.B. (John Boynton) 121

Primrose, Hon Neil 33-4, 51n.126

Probate, Divorce and Admiralty Division of the High Court 180, 209n.145

prostitute $4,21,57,61,64,71,73,80,91 n .14$, 97n.134, 109, 165, 169n.81, 208n.119 'amateur' 5, 6, 74, 97n.134 
Punch 159, 160, 173n.155, 174n.171

Queensberry, Marquis of (John Douglas) $18,51 n .130$

Rattenbury, Alma 211 repression, Freudian ideas of 155 reproduction $26,178,180,203$ respectability $4,19,21,25,73,79,107,114-15$, $124-5,141,181,192,194,198,202$, 206n.70

Reynolds's News/Newspaper 9, 86-9, 115, $144,164,181$

Rhodes, Kathlyn 159, 174n.189

Rich, Paul 71, 96n.106

Robb, George 7, 13n.34, 5on.115, 126n.9, 17 on. 116 with Nancy Erber Disorder in the Court 11n. 5,12 n.24, 128n.57

Roberts, May 85-90, 211, 215, 217

Rohmer, Sax 69, 85, 95n.97

Dope: the Story of Chinatown and the Drug Traffic 83

romance $18,87,106,129$ n.94, 132, 134, 152

Oriental romance 155, 157, 165

see also desert romances

Rothermere, Lord (Harold Harmsworth) 8-9

Rose, Sonya O. 6, 13n.29, 43, 54n.188, 96 n.117

Ross, Robert 31, 41

Rowbotham, Sheila 131n.16o

Russell, Christabel 10, 176-203, 208n.113, n.133, 210, 212-18

Russell, Geoffrey 179, 195, 197, 212

Russell, John 176-203, 204n.25, 206n.72, 208n.113, n.12o, 212

sadism 28-9, 31-2, 37, 50n.95, n.105, 140, 168n.49, 215

Said, Edward 151, 155, 170n.120

Salome 19-23, 26, 29, 32-3, 38

Salome, play by Oscar Wilde 15-16, 18, 29, 31-3, 36-7, 51n.141, 215

'Vision of Salome' dance 19, 21, 46n.27

Sassoon, Siegfried 36, 52n.144

Savage, Gail 199, 209n.139, 209n.147
Savoy Hotel 1, 58, 132-3, 139-40, 143, 166n.6

Searle, G.R. 46n.15, 47n.43, 53n.167

Seed, John 81, 95n.10o, n.102, 96n.104, 98n.144, 99n.171, n.173

sexlessness 194

sexology $17,37,52 \mathrm{n} .150$

sexuality $27-8,144$

female $4,6,10,16,27,90,110,113,158,213$

heterosexuality 216-17

male 72-3

sexual knowledge $26,32,44,178,192-4$, 196, 207n.108

sexual perversion $15,17,26,28-9,31-2$, 35-7, 40, 43-4, 51n.141, 59, 138-41, 148, $153-4,162,165,167 n .43,215-17$

Sheherazade 156

sheik 147, 159, 160, 164, 165, 171n.138, 174n.171, 218

The Sheik 159, 161-3, 174n.168, n.183, 214

shell-shock 2, 117

Siddall, Lilly 73, 97n.131, 98n.145

Simon, Sir John 188, 190, 196, 205n.36

Simon, Maitre Odette 143

Smith, Richard 73, 95n.103, 97n.121

sodomy 37, 153, 171n.138, 215

Solicitor General 104, 110, 117

Sparrow, Gerald 110, 127n.55

Spencer, Harold 18-19, 26, 28, 33, 35, 46n.17, 5on.118

Spilsbury, Dr Bernard 62, 93n.51

spiritualism 189, 207n.88

see also clairvoyant

'spy fever' 38

see also 'hidden hand'

Starling, Ernest 190

Stead, William (W.T.) 50n.95, 63

Stoker. Bram 153

Stopes, Marie 188, 191-2, 206n.72 see also Married Love

Stratford police court 103, 111

suburbs 103, 106, 109, 114-15, 122-5, 129n.89, 212 suburban female 109, 112, 115 suburban male 114

suffrage see vote suffragettes 41, 43

Summerfield, Penny 5, 13n.25

Summerscale, Kate 7, 13n.35 
Sumner Holmes, Anne 111, 127n.56, 169n.91 Sunday Chronicle 9, 27, 78

Sunday Express 9, 60, 63, 64, 71, 111, 119, 134, $158,163,182,197,199,200,214,216$

Sunday newspapers 8, 9, 199

Sunday Pictorial 9, 15, 44

'surplus women' 11n.3

Sutor, Jacob (Jacobus X) 153

Szreter, Simon 207n.108

Tabili, Laura 79, 95n.101, 96n.115, 97n.119, $98 \mathrm{n} .160,99 \mathrm{n} .163$

\section{Tatler 64}

Tennant, Margot see Asquith, Margot Tennyson Jesse, Fryn 211, 218n.5, n.6 Thames Police Court 83, 88

Thane, Pat 96n.115

theatre 19, 27, 47n.33, n.39, 81, 103, 123, 216 trials as $33,119,121,150,155,180,214$

Thom, Deborah 12n.19

Thompson, Edith 1, 10, 102-25, 125n.6, n.7, 126n.11, 127n.32, n.41, 128n.61, n.78, $136,145,147,150,154,160,180,184,196$, 204n.8, 211, 213-14, 217-18

Thompson, Percy 103, 108, 111-15, 117-18, $120,123-4,213$

Thompson, Richard 108-10, 113, 115, 123

Thomson, Basil 36, 51n.145

Times, The 4, 8, 9, 36-9, 43, 57, 73, 78, 87, 121, $161,215-16$

Times Literary Supplement 161

Tinkler, Penny 91n.4

transvestism 49n.93, 125n.2, 184 see also cross-dressing

Travis, Jennifer 16, 45n.10, 46n.13

treachery 9, 17, 23, 43-4, 216-17

trial audience $31,33,66,104,121,149-50$, $156,203,209 n .155$

female 1, 119-20, 134, 154, 165, 182, 214, 218 trial transcripts 2, 9, 10, 140, 178

Tutankhamen 151-2, 155

Ulysses 112

unemployment 2, 70, 110, 120

Union Francaise pour le Suffrage des

Femmes 143

Usborne, Cornelie 11n.13, 206n.82, 207n.88
Valentino, Rudolph 159-61, 174n.17o, n.174 vamp 124, 131n.16o vampires 19, 69, 76 Vaughan, Father Bernard 31 veil $29,107,137,156,164$ venereal disease $5-6,12 \mathrm{n} .23,73,111$ veronal 49n.93, 58, 92n.23

Vigilante, The 15, 17, 22-3, 27, 36-9, 42, 215 Vigilante Society 40

Villiers Stuart, Eileen 26, 33-4, 5on.122

Visram, Rosina $13 n .26$

vote $4,17,41-4,55-7,59,143,146,170$ n.111, 217 Vote, The 122

Wachman, Gay 45n.10, 51n.130

Walkowitz, Judith R. 13n.38, 46n.10, 47n.29, n.34, n.37, n.47. 48n.6o, 5on.95, 52n.153, 92n.43, 93n.56, 94n.66, n.69, 98n.137, $126 \mathrm{n} .13,168 \mathrm{n} .73$

'war babies' 5

Weekly Dispatch 9, 39, 108, 123

Weis, René 125n.6, 126n.10, n.11, n.24, n.29, 127n.33, 43, 47, 128n.59, n.73, 13on.119, 131n.152, n.156, 211, 219n.8

The Well of Loneliness 112

West, Rebecca 65, 122, 124, 230n.105

Western Mail 71, 110

Westminster Coroner Court 58

Westminster Gazette 35

White, Arnold 17, 39, 40, 43, 45n.4, 52n.162, 53n.17o, n.181

Whiteley, Cecil 116

Wickham, Gilbert 102-3, 119, 124

Wilde, Oscar 15-16, 18, 22, 29, 32-3, 41, 44, 46n.21, 51n.131, 53n.170, 136, 153

Williams, Ivy 136

Witchard, Anne 73, 95n.97, 97n.123, n.126, n.129, n.130, 99n.179

women's patrols and women police 5, 44, 911.14 Woollocott, Angela 12n.22, 98n.134

Woolf, Virginia 1, 22

World's Pictorial News 107, 118, 123, 212

Young, Filson 14n.48, 104, 116, 125n.7, 126n.12, 127n.51, 128n.61, n.69, n.71, n.75, n.76, 129n.10o, 130n.108, n.111, n.115, 204n.8, 213, 219n.18 